\title{
Crystallization and vitrification of semiflexible living polymers: A lattice model
}

\author{
Gautam I. Menon \\ Department of Physics, Simon Fraser University, Burnaby, British Columbia, Canada V5A 1S6 \\ Rahul Pandit* \\ Department of Physics, Indian Institute of Science, Bangalore 560012 , India
}

\begin{abstract}
We study the systematics of a $d$-dimensional lattice model for melts of semiflexible living polymers. For $d=2$ and 3 our model, which includes vacancies, loops, and the possibilities of polymerization and polydispersity, exhibits both equilibrium crystallization and glass formation in the wake of a quench. We study these analytically, in some limits, and via extensive Monte Carlo simulations. A continuous Ising-type transition separates crystalline and disordered phases for the $d=2$ square lattice. If loop formation is favored in $d=2$, crossover effects lead to power-law decays of polymer-length distributions over large length scales, strong fluctuations in thermodynamic quantities, and slow relaxation. These crossover effects arise because of the proximity of a phase with an infinite correlation length in one limit of our model. For the $d=3$ simple cubic lattice our model has a first-order crystallization transition. Quenches from the disordered to the ordered phase yield glassy, metastable configurations for both $d=2$ and 3. We study the latter case in detail and find logarithmically slow relaxation out of these metastable configurations, a frustration-driven glass-crystal transition, and an exotic lamellar glass. We propose a Monte Carlo analog of scanning calorimetry and use it to study these glasses. We discuss the relevance of our work to experiments on different systems of living polymers, earlier studies of crystallization in polymeric melts, and some theories of the glass transition in model systems. [S1063-651X(99)07201-3]
\end{abstract}

\section{INTRODUCTION}

The crystallization and vitrification of polymer melts has been of considerable interest since the early work of Flory [1] and Gibbs and DiMarzio [2]. Crystalline phases may be seen in a variety of long-chain systems at low temperatures [3], such as poly(thio-11,4-phynylene) and polystyrene [4], as well as in some biological macromolecules with intrinsic stiffness, e.g., DNA, and some synthetic polypeptides. Crystallization occurs when a polymeric melt is cooled very slowly; however, more often than not, a polymeric glass or an entangled gel forms given typical laboratory cooling rates. Crystallization can also occur via steric repulsion if the concentration is increased sufficiently. The crystallization transition is first order in three dimensions; we know of no experiments on such crystallization in two dimensions. The scope of studies of polymer solidification has been enlarged by the recognition that some systems contain living polymers, i.e., polymers whose lengths fluctuate and attain an equilibrium length distribution at any given temperature $T$. The threadlike micelles that form in some water-surfactant systems [5] are an example of living polymers. Dilute systems of such threadlike micelles were studied first [6]; the dense systems [7] being investigated now have shown viscoelastic, glassy states (e.g., in the water-cetyltrimethylammonium bromide-sodium 3-hydroxynaphthalene-2carboxylate system [8]). It has been suggested recently [9] that liquid sulfur [10] and selenium [11], poly

\footnotetext{
*Also at Jawaharlal Nehru Centre for Advanced Scientific Research, Bangalore, India.
}

( $\alpha$-methylstyrene) [12,13], and protein filaments [14] are also assemblies of living polymers. These living polymers are semiflexible: It costs energy to bend them, so at low $T$ they straighten, thereby favoring the formation of ordered phases, but, as noted above, entangled gels or glasses form more readily. In this paper we present a systematic study of crystallization and vitrification in the model for semiflexible, living polymers introduced by Menon, Pandit, and Barma [15]. Some of our results on crystallization in $d=2$ and on glass formation in $d=3$ have been reported briefly earlier $[15,16]$. We also discuss the relation of our work with that of other groups for the crystallization of polymer melts $[1,9,17-24]$ and its implications for glass formation in general [25-27] and in the polymer context [2,28,29].

The statistical mechanics of polymeric melts is a complex problem, so most authors [9,15-24] have followed Flory [1] and used lattice models to study it. Despite this simplification and considerable theoretical effort over the past four decades, many features of the behavior of such lattice models remain unclear: e.g., there is little consensus [15,18,19,22] regarding the nature of the transition in the Flory model in $d=2$. Numerical studies of glass formation in lattice models for polymeric melts have been attempted only recently $[16,28,29]$. Our two main goals have been (i) to develop and study a lattice model for melts of semiflexible, living polymers that is a natural extension of Flory's model [1] for conventional polymer melts and (ii) to use our study to resolve some of the controversies surrounding the nature of the crystallization transition in Flory's model.

In early work with Barma [15] we developed a twodimensional lattice model for melts of living polymers; we have now generalized it to $d=3$. Our model is akin to Flo- 
ry's [1] in that it is defined on the links (not vertices as in Refs. $[9,23,24]$ ) of a $d$-dimensional hypercubic lattice, builds in semiflexibility via an energy cost $\epsilon$ for right-angle bends, and enforces the self-avoidance of chains. It generalizes Flory's model by allowing for vacancies, controlled by a chemical potential $\mu$, and scission and fusion of chains, controlled by an energy cost $h$ for open ends. Thus our model allows for polymerization, chain polydispersity, and ring polymers. Furthermore, as we will show, it yields an equilibrium polymer-length distribution at any $T$, i.e., we get a system of living polymers.

Our study yields a variety of interesting results for (i) the nature of crystallization in our model polymeric melt, (ii) a possible reason for the discrepancies between earlier studies [1,17-23], and (iii) glass formation in our model. We begin with a qualitative summary of these results: In our model the crystallization transition is continuous in $d=2$ (Ising type on the square lattice). In some regions of the phase diagram correlation lengths are very large because of the proximity of the high- $T$, power-law phase of the $F$ model $[17,22,30]$, which obtains in one limit of our model; it leads to a powerlaw decay of polymer-length distributions over intermediate length scales (less than $L_{c}$, a crossover length that we estimate) and near-critical behavior. The resulting slow equilibration accounts, we believe, for the discrepancies [1,17$19,22,23$ ] between earlier studies of $d=2$ lattice models for semiflexible polymeric melts (see Sec. V). In $d=3$ our model has a first-order transition: The order parameters (see below), internal energy, and mean polymer size are discontinuous at the transition, in agreement with Flory's theory [1]. At low $T$ the average chain length $\langle\ell\rangle \sim L$, the linear size of the system; we argue, however, that, for sufficiently large $L,\langle\emptyset\rangle$ saturates to an $L$-independent constant that depends on $T, \mu$, etc., for both $d=2$ and 3. Only at $T=0$ do we get $\langle\ell\rangle \sim L$. Slow relaxation out of metastable states is common in $d=2$ and 3: If the melt is quenched rapidly from high to low $T$, disordered metastable states obtain. In $d=3$ they are completely disordered [Fig. 1(a)] if the open-end cost $h$ is large or partially ordered lamellar glasses [the disordered stacking of ordered layers in Fig. 1(b)] for intermediate $h$. In the former case (large $h$ ) relaxation is logarithmically slow whereas in the latter (intermediate $h$ ) quenched configurations evolve into a lamellar glass over a time $\tau_{l g}$ $\left[\sim \exp (2 \beta / h)\right.$, where $\beta \equiv 1 / T$ (Boltzmann constant $\left.\left.k_{B}=1\right)\right]$. Furthermore, the system falls out of equilibrium as it is cooled at a finite rate and the metastable states behave like real polymeric glasses $[3,4]$ when studied by a Monte Carlo analog of scanning calorimetry, which we have introduced recently [16]. Order-parameter autocorrelation functions are slowly decaying exponentials for shallow quenches, but for deeper ones these decays are too slow to obtain reliable fits. Most interesting of all, we find that lowering $h$ eases the frustration in the disordered network obtained on quenching, thereby inducing an apparently continuous glass-crystal transition, but one that is not related to an underlying equilibrium phase transition.

The calculations that lead to the results summarized above are described in the remaining part of this paper, which is organized as follows. In Sec. I we give a brief overview of the Flory [1] and related models, comment on their connection with our work, and end with a description of our model.
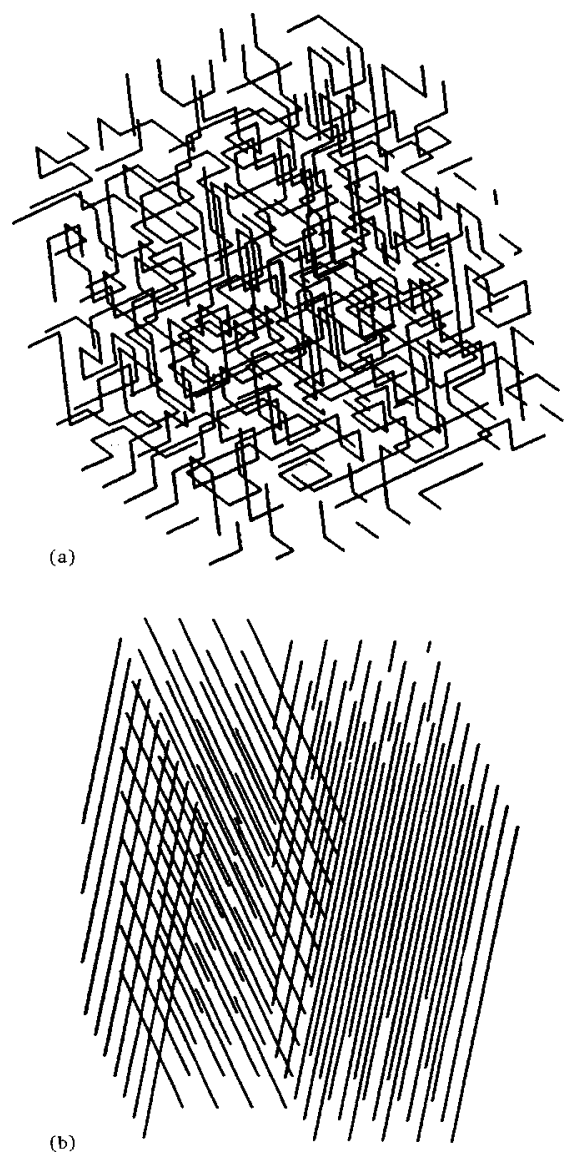

FIG. 1. Configurations of polymers in disordered glassy states of our model in $d=3$ obtained from instantaneous snapshots after a quench (see the text) in our simulations at (a) large $h(=3.5$ here) and (b) intermediate $h(=1.5$ here).

Sections II and III deal with the equilibrium properties of our model in $d=2$ and 3, respectively. Section IV is devoted to a study of glass formation in our model principally in $d$ $=3$. Section $\mathrm{V}$ analyses our results in the light of earlier work on related models and comments on the possibility of experimental studies of our predictions.

\section{MODELS}

Theoretical studies of the crystallization of melts of semiflexible polymers trace their origins to the pioneering work of Flory. In his model [1] self-avoiding polymer chains of fixed length are placed on the links of a square or simple cubic lattice. Gauche configurations, i.e., right-angle bends in the chain, cost an energy $\epsilon$; trans configurations with no bends cost no energy. Flory's mean-field approximation [1] for this model uses an ansatz for the probability that a specified link is vacant after a certain number of chains have been laid down on the lattice. This yields a first-order transition for all $d$. At low $T(\Sigma \epsilon)$ the chains straighten and, for a dense packing, align along the $x$ or $y$ (or $z$ in $d=3$ ) axes. This approximation yields essentially complete order in the low- $T$ phase. The transition occurs because of intramolecular interactions, parametrized by $\epsilon$, and self-avoidance (which is the only way in which intermolecular interactions enter the model).

Despite the success of the Flory theory in predicting that 


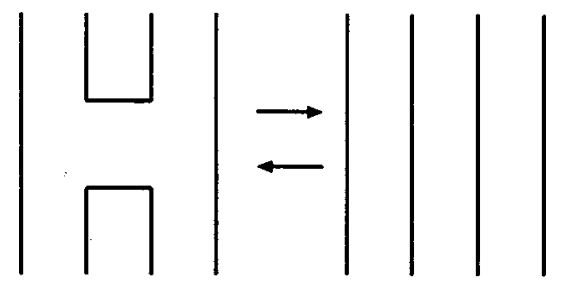

FIG. 2. Schematic illustration of a defect configuration in our two-dimensional polymer model (the $\mathbb{H}$-shaped configuration of links). Such defects, which cost energy $4 \epsilon$, are entropically favored at low $T$. Our multilink move (illustrated above) converts this configuration of links to an aligned one. To satisfy detailed balance the reverse move is also attempted with probability $\exp (-4 \beta \epsilon)$.

polymer crystallization is a first-order transition, several doubts remained about its validity, particularly in low dimensions. In an important paper, Gujrati and Goldstein [18] demonstrated the failure of Flory's mean-field approximation explicitly in the limit where a single, semiflexible, selfavoiding chain visits all sites of a square lattice (i.e., it executes a Hamilton walk). They did this by bounding the number of walks with a specified density of bends and thus showed that the entropy $s(T)>0$ for temperatures $T>0$. Gujrati and Goldstein [18] noted that defect configurations of the type shown in Fig. 2 tend to disorder the ground state and they used them in the construction of their bound. The energy cost for such a defect is $4 \epsilon$, but, once it is created, it is free to slide in either the $x$ or $y$ direction (in $d=2$ ). Thus these defects are entropically favored and they clearly reduce the perfect crystalline ordering predicted by the Flory approximation at low temperatures.

Baumgärtner [19] and later Baumgärtner and Yoon [21] used Monte Carlo simulations of lattice models for semiflexible polymers in $d=2$ and 3 to obtain first-order transitions in a dense system of infintely long chains (i.e., for which the ratio of the chain lengths to the system size $L$ goes to a constant as $L \rightarrow \infty$ ); a system with chains of finite size showed no transition. They used a reptation algorithm [31] with fixed chain lengths and a fixed fraction of empty sites.

Saleur [22] mapped configurations of the $F$ model [30] onto polymer configurations on a square lattice to suggest that, for $d=2$, the Flory model has an infinite-order, $F$-model-type transition. However, the polymer analog of the $F$ model admits no open ends (i.e., all polymers form rings or loops); moreover, it allows for polydispersity (unlike the original Flory model, which admits only open chains with a fixed number of monomers). Vacancies (sites with no incoming occupied bonds) are also excluded in the $F$-model mapping. Saleur was able to remove some of these constraints in his transfer-matrix calculation; in particular, loops could be forbidden and his technique allowed for the introduction of a small number of vacancies. However, we will prove exactly (Sec. II) that the introduction of such vacancies (which leads to a special 7-vertex model) yields a phase with a finite correlation length, so it cannot be like the high-temperature phase of the $F$ model, which has an infinite correlation length.

Other simulations have concentrated on independentmonomer-state (IMS) models $[9,23,24]$ in which site configurations (truncated link configurations at vertices) attach to form polymer chains. These models clearly describe melts
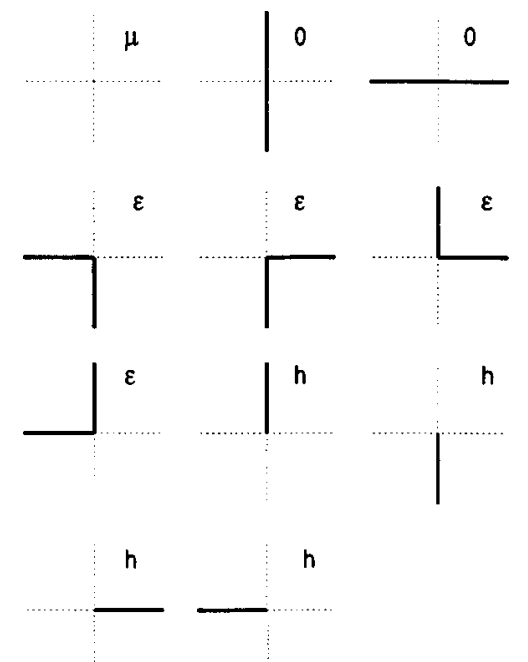

FIG. 3. Vertex configurations and their energies for our twodimensional model. Full lines indicate occupied links and dashed lines unoccupied links.

of living polymers, as chain lengths are not constrained to be fixed. There are, however, important differences between our model and the IMS models. In our model the dynamical variables are bonds placed on the links of a lattice, whereas the IMS models are site models. As a consequence, IMS models admit configurations that are not present in our model. We compare their results with ours in Sec. V.

In our $d$-dimensional model, defined on the links of a hypercubic lattice, an occupied link represents a monomer. These monomers can fuse to form polymers, which are selfavoiding (branching is forbidden). Straight segments of a polymer (trans configurations) cost no energy, but rightangle bends (gauche configurations) cost an energy $\epsilon$. The energy for an open end is $h$ and for a vacancy (a vertex surrounded by four unoccupied links) $\mu$. We set the energy scale by choosing $\epsilon=1$. The vertex configurations allowed in $d=2$ are shown in Fig. 3 together with their energies. In most of our two-dimensional studies monomers on different links do not interact except via self-avoidance as in Flory's model [1]; however, in some of our studies we have included some interchain interaction (see below). In $d=3$, if we do not have both intra- and interchain interactions, the ground state turns out to be infinitely degenerate [20]. Thus we associate an attractive energy $J$ with a pair of parallel, occupied links that are part of the same plaquette. An attractive energy of this type is often used to approximate the attractive part of a van der Waals interaction. We will describe our Monte Carlo procedure later, but we note here that we do not control the density of monomers. It achieves an equilibrium value that depends on $h, \mu$, and $T$ since we use the grandcanonical ensemble rather than the fixed-chain-length canonical ensemble used in reptation simulations [19,21,31].

\section{TWO DIMENSIONS}

The $T=0$ phase diagram of our model in $d=2$ contains three distinct phases separated by first-order phase boundaries. These boundaries are (a) $\mu=h$ with $h \leqslant 0$, separating vacancy (no links occupied) and dimer phases (with a powerlaw decay of correlations [32]); (b) $\mu=0$ with $h \geqslant 0$, sepa- 


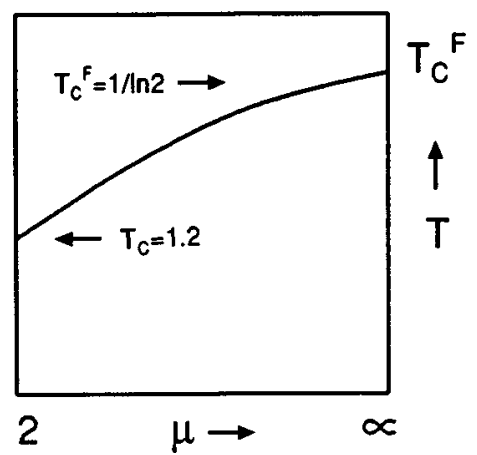

FIG. 4. Schematic phase diagram in the $\mu-T$ plane for our model in two dimensions and with $h=\infty$. $T_{C}^{F}$ indicates the transition temperature in the $F$-model limit $\mu=\infty$. At $\mu=2$ the transition temperature $T_{c} \simeq 1.2$. The line separating the high-temperature disordered phase from the low-temperature ordered one indicates a continuous transition, which is of the two-dimensional Ising type for the region shown except at $\mu=\infty$, where it is of the $F$-model type.

rating vacancy and ordered phases; and (c) $h=0$ with $\mu$ $\geqslant 0$, separating dimer and vacancy phases [33]. Figure 4 shows a schematic phase diagram for $d=2$ in the $\mu-T$ plane for $h=\infty$; the topology of this phase diagram is similar for $h \gg \epsilon$. One might have expected a Blume-Capel-type [34] phase diagram, but we have found no first-order transition in the range of parameter values we study; it is possible, though, that the tricritical point occurs either at or very close to $T=0$. The Ising transition that separates our ordered and disordered phases remains if monomers on next-nearestneighbor parallel links attract each other with an energy $J$.

We have drawn the schematic phase diagram of Fig. 4 on the basis of extensive Monte Carlo simulations principally in the regimes $\mu, h>0$, with either $h \gg \epsilon$ or $h \simeq \epsilon$ with $\mu$ constant (see below). These simulations are augmented by a mapping onto the $F$ model [30] at $\mu=h=\infty$ and an exact solution (by a mapping onto a free-fermion model $[35,36]$ ) for $h=\infty$ and $T=2 \epsilon / \ln 2$; in particular, we show that our model has a finite correlation length for these parameter values if $0<\mu<\infty$.

At $\mu=h=\infty$ (no vacancies or open ends) the configurations of our model can be mapped onto those of the $F$ model $[17,22,30]$, which has a high-temperature phase with a power-law decay of correlation functions and a lowtemperature, ordered phase [all polymer links aligned in the same $(x$ or $y$ ) direction at $T=0]$. We show that the hightemperature, power-law phase is destroyed on the introduction of an arbitrarily small amount of vacancies and/or open ends. However, if the density of open ends is small, the properties of this phase are manifested in strong crossover and slow-equilibration effects. If the number of open ends is strictly zero, the correlation length scales exponentially with $\mu$. We prove this explicitly for $T=2 \epsilon / \ln 2$ and believe that this result should hold in the high-temperature phase in general. At high temperatures our model has a conventional disordered phase. Crossover from the $F$-model, power-law phase to a conventional disordered phase appears to be governed principally by the energy cost for open ends $h$, when both $h$ and $\mu$ are finite and $h \gg \mu$. We show that, on the square lattice, the transition from the ordered to the disor-

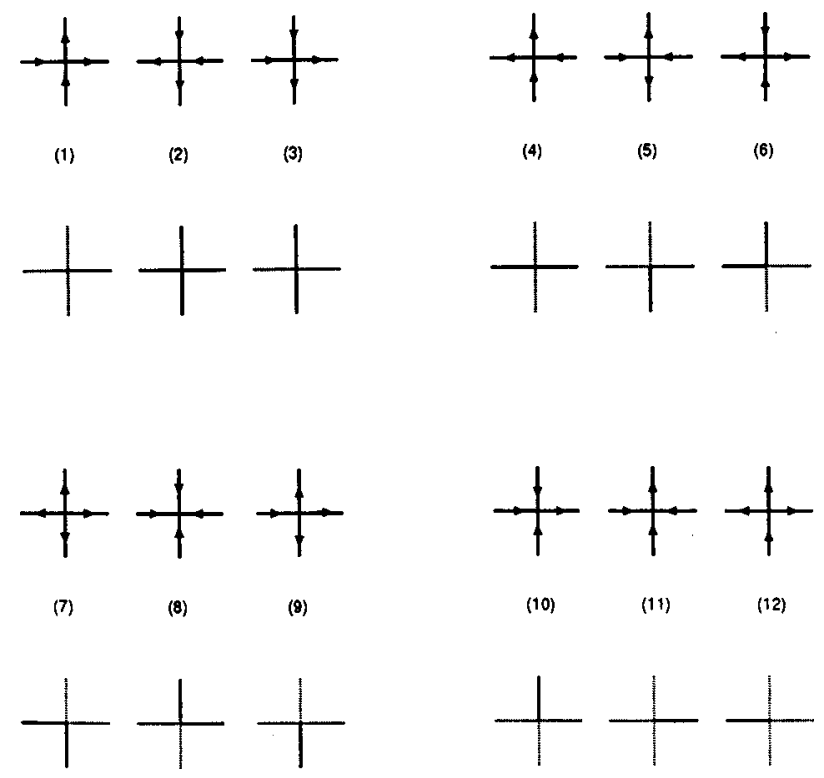

FIG. 5. Our polymer model is equivalent to an 11-vertex model in two dimensions. This figure shows how configurations in these models map onto each other. Twelve vertices are shown; however, vertex 2 is not allowed in our model (self-avoidance).

dered phase is in the $d=2$ Ising universality class when the density of open ends is large. We argue that the transition, when continuous, should belong to this universality class except at isolated points such as the $F$-model limit. However, our numerical data are not good enough to prove this conclusively in the crossover regime.

\section{A. Exactly solvable limits}

The link configurations in our two-dimensional model can be mapped exactly onto an 11-vertex model on a square lattice, specified by associating arrows with the bonds (and preserving the directions of the arrows when vertices are put together). If the weights $w_{i}$ are assigned to each allowed vertex, the partition function

$$
Z=\Sigma w_{1}^{n_{1}}, \ldots, w_{11}^{n_{11}}
$$

where the summation is over all topologically allowed configurations and $n_{i}$ is the number of times vertex $i$ occurs in a configuration. One such mapping between polymers and vertex models is illustrated in Fig. 5: We choose a basic vertex configuration (vertex 1, say) and associate bonds with those arrows of vertices $1-11$ that are directed in the sense opposite to the corresponding arrow in the basic configuration. The polymer configurations thus generated are then all allowed if we restrict ourselves to vertices 1 and 3-11 (selfavoidance is ensured by the elimination of vertex 2).

If open ends, vacancies, and trivalent and tetravalent vertices are forbidden, the vertex model does not have vertices 1, 2, and 9-11 (Fig. 5), so it is a 6-vertex model. By using the symmetries of the symmetric 8-vertex model [37], defined via vertices $1-8$, this model can be mapped onto the $F$ model, solved by Lieb and $\mathrm{Wu}$ [30], and conventionally defined by using vertices $1-6$. If we assign weights as shown in Fig. 5 and make the (symmetric) choice 


$$
\begin{aligned}
& w_{1}=w_{2} \equiv u_{1}, \quad w_{3}=w_{4} \equiv u_{2}, \\
& w_{5}=w_{6} \equiv u_{3}, \quad w_{7}=w_{8} \equiv u_{4},
\end{aligned}
$$

then the following conditions hold [36]:

$$
\begin{aligned}
& Z\left(u_{1}, u_{2} ; u_{3}, u_{4}\right)=Z\left(u_{3}, u_{4} ; u_{1}, u_{2}\right), \\
& Z\left(u_{1}, u_{2} ; u_{3}, u_{4}\right)=Z\left(u_{2}, u_{1} ; u_{4}, u_{3}\right), \\
& Z\left(u_{1}, u_{2} ; u_{3}, u_{4}\right)=Z\left(u_{2}, u_{1} ; u_{3}, u_{4}\right) .
\end{aligned}
$$

For the $F$ model

$$
u_{1}=\exp (-\beta \epsilon), \quad u_{2}=\exp (-\beta \epsilon), \quad u_{3}=1, \quad u_{4}=0
$$

We use the symmetry properties (3) to obtain

$$
Z\left(u_{1}, u_{2} ; u_{3}, u_{4}\right)=Z\left(u_{3}, u_{4} ; u_{1}, u_{2}\right)=Z\left(u_{4}, u_{3} ; u_{1}, u_{2}\right)
$$

and thence the equivalence of the $F$ model and our polymer model with no vacancies or open ends (all polymer bends cost an energy $\epsilon$ ). The $F$ model has an infinite-order phase transition at $T=T_{c F}=\epsilon / \ln 2$ and there is a power-law decay of correlations in its high-temperature phase, as in the lowtemperature phase of the two-dimensional $X Y$ model. The exponents governing this decay vary continuously with $T$.

If the vertex weights in the 8-vertex model satisfy

$$
w_{1} w_{2}+w_{3} w_{4}=w_{5} w_{6}+w_{7} w_{8}
$$

the model is again solvable since it becomes the free-fermion model of Fan and $\mathrm{Wu}$ [36]. We choose parameters so that the free-fermion condition is satisfied and the tetravalent vertex is eliminated:

$$
\begin{gathered}
w_{1}=\exp (-\beta \mu), \quad w_{2}=0, \quad w_{3}=1, \\
w_{4}=1, \quad w_{5}, \ldots, \quad w_{8}=\exp (-\beta \epsilon),
\end{gathered}
$$

with $T=2 \epsilon / \ln (2)$; this vertex model is equivalent to our polymer model with bends and vacancies (but no open ends) at a fixed temperature $T=2 T_{c F}$. The free energy (per vertex) in the free-fermion limit [36] is

$$
\begin{aligned}
-\beta \mathcal{F}= & \frac{1}{8 \pi^{2}} \int_{-\pi}^{\pi} d \theta \int_{-\pi}^{\pi} d \phi \ln [2 a-2 b \cos \theta-2 c \cos \phi \\
& +2 d \cos (\theta-\phi)+2 e \cos (\theta+\phi)]
\end{aligned}
$$

with

$$
\begin{gathered}
2 a=w_{1}^{2}+w_{2}^{2}+w_{3}^{2}+w_{4}^{2}, \\
b=w_{1} w_{3}-w_{2} w_{4}, \\
c=w_{1} w_{4}-w_{2} w_{3}, \\
d=w_{3} w_{4}-w_{7} w_{8}, \\
e=w_{3} w_{4}-w_{5} w_{6},
\end{gathered}
$$

so, given our choice of vertex weights, the free energy of our model is

$$
\begin{aligned}
-\beta \mathcal{F}= & \frac{1}{8 \pi^{2}} \int_{-\pi}^{\pi} d \theta \int_{-\pi}^{\pi} d \phi \ln \left[y^{2}+2-2 y \cos \theta-2 y \cos \phi\right. \\
& \left.+2\left(1-x^{2}\right) \cos (\theta-\phi)+2\left(1-x^{2}\right) \cos (\theta+\phi)\right],
\end{aligned}
$$

where $x=\exp (-\beta \epsilon)$ and $y=\exp (-\beta \mu)$.

To analyze the long-distance behaviors of correlation functions we find the minimum value of the argument of the logarithm in Eq. (10). If it vanishes the phase is massless; otherwise there is a finite correlation length. This follows from the structure of the Grassmann representation for the correlation functions for the free-fermion model $[35,40]$. The correlation length is infinite for $y=0$ (the $F$-model limit), as expected, but finite for any nonzero concentration of vacancies. The result in the $F$-model limit is in accordance with a result derived by Baxter at the $2 T_{c F}$ point [38]. We find that

$$
\xi \sim e^{\mu / \epsilon \ln 2},
$$

where $\xi$ is the correlation length. If $\xi \gg L$ (which happens when $\mu$ is large), crossover behavior results. Whereas this exact result demonstrates the existence of a finite correlation length in the limit of no open ends and a finite concentration of vacancies, it tells us nothing about the behavior if a finite concentration of open ends is present. However, we believe, on the basis of our simulations (see below) that this crossover behavior persists as long as the density of open ends is small. In particular, our exact result differs from earlier work by Saleur [22], which suggests that the Flory model with a finite concentration of vacancies continues to have $F$-modeltype behavior.

\section{B. Simulations}

In our simulations we used square lattices of linear size $L$ ranging from 4 to 80 , with periodic boundary conditions. In most cases, we performed $\left(5 \times 10^{5}\right)-\left(7.5 \times 10^{5}\right)$ Monte Carlo steps (MCS) per link at each set of values of $T, h$, and $\mu$; in some cases we went up to $10^{6}$ MCS. We used the algorithm of Metropolis et al. [39] and single-link moves in which a link update was attempted (by the removal of a bond if one were initially present or by its addition if not forbidden by self-avoidance). We went through the lattice sequentially. Every $50 \mathrm{MCS}$, we also used multilink moves in which a defect [18] of the type shown in Fig. 2 was replaced by a configuration with all links parallel or vice versa, with a probability $\exp \left(-4 \epsilon / k_{B} T\right)$ in order to ensure detailed balance; we found that such multilink moves were essential for equilibration at low $T$. Typically we discarded the first $10^{5}$ MCS before accumulating data for thermodynamic functions (which we did every 50 MCS per link). Convergence was checked by tracking the energy per link every 1000 iterations; convergence to one part in $10^{3}$ or better was attained.

We computed the internal energy $U$, the specific heat $C$, $N_{y}$ and $N_{x}$, the numbers of occupied links in the $y$ and $x$ directions, respectively, $N_{\text {links }}$, the number of links occupied in the fully ordered state, and thence the order parameter 


$$
M=\left(N_{y}-N_{x}\right) / N_{\text {links }}
$$

and the order-parameter susceptibility

$$
\chi=\frac{1}{T} \frac{\left\langle\left(N_{y}-N_{x}\right)^{2}\right\rangle-\left\langle\left(N_{y}-N_{x}\right)\right\rangle^{2}}{2 N_{\text {links }}} .
$$

We also obtained the mean numbers of vacancies $N_{v}$, bends $N_{b}$, open ends $N_{o}$, the normalized fourth moment or kurtosis $S_{4}=\left\langle M^{4}\right\rangle /\left\langle M^{2}\right\rangle^{2}$, and $P(\ell)$, the distribution of polymer lengths $\ell$, as functions of $T, h, \mu$, and $L$. The thermodynamic quantities plotted in subsequent figures (except for the distribution of polymer lengths) are normalized by the number of links ( $=2 L^{2}$ in $\left.d=2\right)$. At the end of this section we discuss the effect of a small interchain, attractive interaction $J$ between parallel monomers on next-nearest-neighbor links.

We performed simulations over a range of parameter values, but concentrated, for definiteness, on two cases in $d$ $=2$ : (a) $\mu=2, h=1.2$ and (b) $\mu=2, h=4$, which lie, respectively, in the regimes $h \simeq \epsilon$ and $h \gg \epsilon$ mentioned above. The results for intermediate parameter values lie between these two limits.

Figures 6(a) and 6(b) [cases (a) and (b), respectively] show $M$ versus $T$ for $L=6,12$, and 20. In Fig. 6(a) error bars are comparable to the sizes of the symbols, but in Fig. 6(b) they are greater (by a factor of 4-5). The transition in case (a) is clearly continuous, but in case (b) such an identification is problematic. Finite-size effects are more clearly visible in Fig. 6(a) than in Fig. 6(b). The considerable fluctuations seen in case (b) are indicative of large correlation lengths, which lead to large correlation times.

Figures 6(c) and 6(d) [cases (a) and (b), respectively] show $S_{4}$ versus $T$ for $L=6,12$, and 20 . The curves for different values of $L$ cross at a single point in Fig. 6(c), so we identify the transition here to be a conventional, secondorder one. A finite-size-scaling analysis of our data (see below) yields $d=2$ Ising exponents. Figure 6(d) is considerably different from Fig. 6(c) insofar as curves for different values of $L$ overlap, within error bars, over a finite range of $T$. Such overlapping normally indicates a power-law phase, in which some correlation length is infinite over a finite region of parameter space. However, it can also occur if the correlation length is very large (but finite) and much greater than $L$. As our free-fermion solution indicates, correlation lengths can be very large over a substantial region of parameter space, leading to signatures that could suggest a powerlaw phase (given simulations for small $L$ ). Our simulations find similar effects away from the free-fermion limit: Even though $h$ is finite, our data are consistent with large correlations lengths and times.

In Figs. 6(e) and 6(f) we show how $N_{v}, N_{b}$, and $N_{o}$ vary with $T$ for cases (a) and (b), respectively. The vacancy concentration $N_{v}$ is small in both cases, so we have a dense melt. In case (a), the density of open ends $N_{o}$ is much larger than in case (b) because the formation of ring polymers is favored in the latter. We expect that $N_{v}, N_{b}$, and $N_{o}$ all inherit the weak nonanalyticity of the energy density at the continuous transition, though we have not checked this explicitly.

In Figs. 7(a) and 7(b) we plot $C$ versus $T$. For case (a) [Fig. 7(a)] its divergence at $T_{c}$ in the $L \rightarrow \infty$ limit shows up clearly. We find the peak height increases as $\log L$, as it should for a transition in the $d=2$ Ising universality class. In case (b) $[$ Fig. $7($ b) $]$ the peak height seems to saturate at a finite value with increasing $L$; however, the data are extremely noisy. (Recall that the $F$-model specific heat [30] shows a similar peak at $T=\epsilon>T_{c F}$.)

In Figs. 7(c) and 7(d) we plot $\chi$ versus $T$ [Eq. (4)]. In case (a) [Fig. 7(c)] as $T \rightarrow T_{c}, \chi \rightarrow \infty$ in the thermodynamic limit. A finite-size scaling plot for this case (Fig. 8) yields $d=2$ Ising exponents. In case (b) [Fig. 7(d)], the noise in our data prevented us from verifying Ising-type scaling at the transition. This noise in the disordered phase of our model arises because of the proximity of the high-temperature, power-law phase in the $F$-model limit of our model. The $F$-model ana$\log$ of our susceptibility is a staggered susceptibility associated with the two types of vertices that appear in the ground state. (These vertices are arranged antiferroelectrically: Arrows on succesive columns are oppositely directed.) This staggered susceptibility is infinite in the range $T_{c F}-2 T_{c F}$ since the associated correlations decay algebraically (with an exponent that increases from 1 at $T_{c F}$ to 2 at $2 T_{c F}$ and finally to 3 at $T=\infty[22,41])$.

In Fig. 9 we plot the angle-averaged correlation function for two links along the $x$ direction, $\rho_{x x}\left(x, y, x^{\prime}, y^{\prime}\right)$ $\equiv\left\langle\rho_{x}(x, y) \rho_{x}\left(x^{\prime}, y^{\prime}\right)\right\rangle-\left\langle\rho_{x}(x, y)\right\rangle\left\langle\rho_{x}\left(x^{\prime}, y^{\prime}\right)\right\rangle$, versus the radial coordinate $r$, for $h=1,2,3$, and 4 . This (connected) correlation function is obtained by averaging over all $x$ links a fixed radial distance away from an $x$ link at the origin and then averaging over all possible choices of this origin. This correlation function is short ranged for small $h$ but decays more and more slowly with increasing $h$. Thus, as we had anticipated earlier, the correlation length can become very large as $h$ increases, until it diverges in the $F$-model limit of our model.

Our results for the distribution of polymer lengths $P(\ell)$ versus $\ell$ are shown in Figs. 10(a)-10(d) [case (a) in Figs. 10(a) and 10(d) and (b) in Figs. 10(b) and 10(c), respectively] for different values of $T$ and $L=20$. In case (a), $P(\ell)$ is clearly exponential [Fig. 10(a)] in the disordered phase; in the ordered phase the envelope of $P(\ell)$ also decays exponentially, but peaks appear at values of $\ell$ commensurate with $L$ at sufficiently low $T$ because of the formation of ring polymers that wind around the system (once or many times since we use periodic boundary conditions). In case (b) our results are as follows: The exponential tail of $P(\ell)$ seen in case (a) occurs only at sufficiently high $T$ [Fig. 10(b)]. At low $T$ and for small $\ell$ ring formation is favored (with an even number of monomers) as there is a large energy cost $h$ for open ends. As a consequence, polymers with an even number of monomers are distributed differently from polymers with an odd number of monomers [Fig. 10(c)]; in our simulations we observe a clear distinction in the scaling behavior of polymer lengths in these two cases. We find that the distribution for chains with an even number of monomers scales as

$$
P(\ell) \sim \ell^{-\vartheta},
$$

where the exponent $\vartheta$ depends on $T, h$, and $\mu$; this powerlaw form holds only if $\ell$ is smaller than a crossover length estimated below. For polymer chains with an odd number of monomers we find that $P(\ell)$ is nearly independent of $\ell$. A 

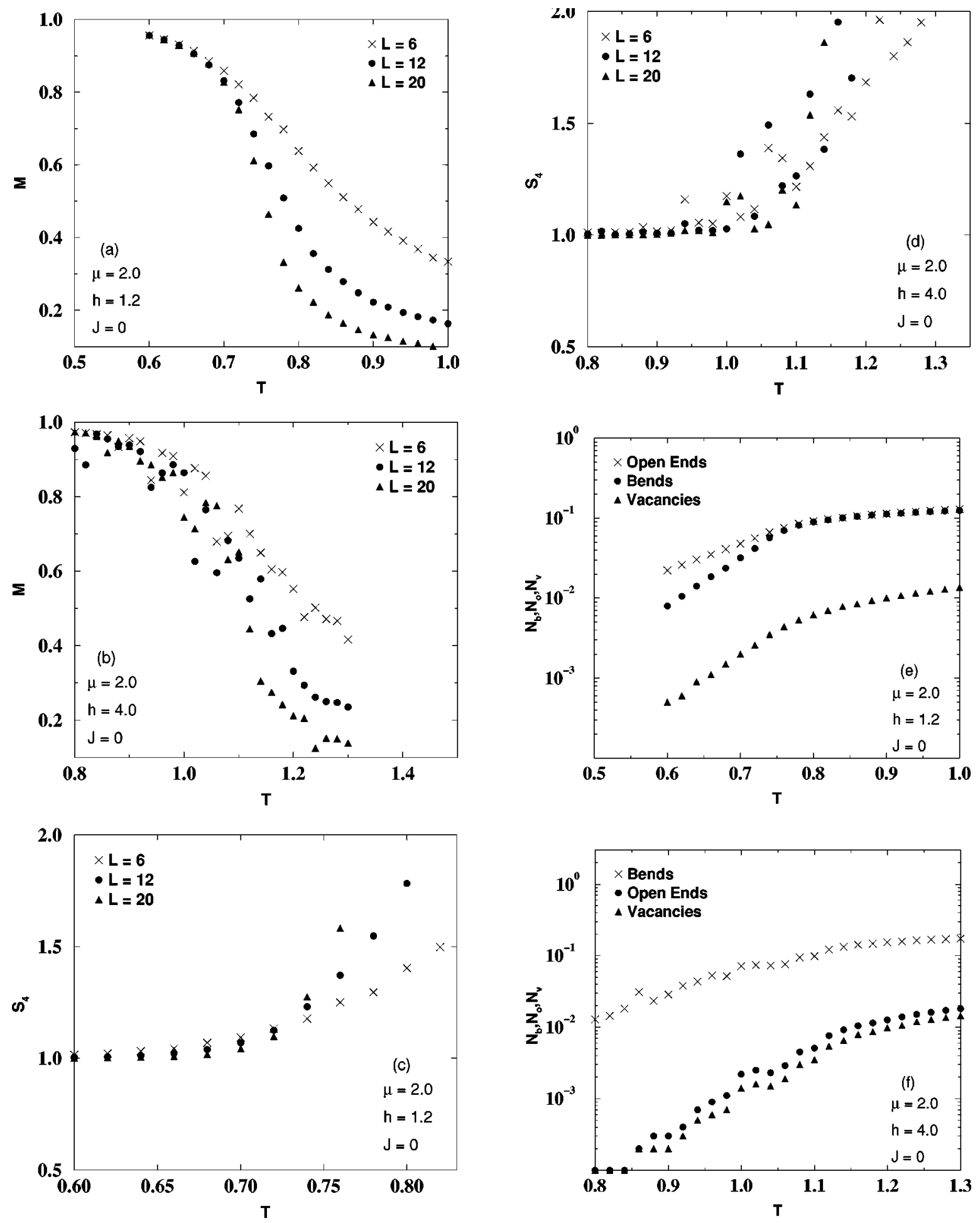

FIG. 6. Order parameter $M$ versus $T$ for $d=2$ as $T$ crosses the transition temperature $T_{c}$. Data are shown for three different (linear) system sizes $L=6,12$, and 20 for (a) $\mu=2, h=1.2$, and $J=0 \quad\left(T_{c} \simeq 0.73\right.$ here) and (b) $\mu=2, h=4$, and $J=0$ ( $T_{c}$ is hard to pinpoint here). The normalized fourth moment $S_{4}=\left\langle M^{4}\right\rangle /\left\langle M^{2}\right\rangle^{2}$ versus $T$ for $d=2$ is shown in (c) and (d) for the same parameters as in (a) and (b), respectively. $N_{v}, N_{o}$, and $N_{b}$, the densities of vacancies, open ends, and bends, versus $T$ for $d=2$ as $T$ crosses the transition temperature are shown in (e) and (f). The parameters in (e) and (f) are the same as in (a) and (b), respectively.

qualitative interpretation of this result is that, in the limit of a small number of open ends (i.e., $h \rightarrow \infty$ ), the formation of open chains occurs by the breaking of rings. Since a ring of length $\ell$ can be broken in $\ell$ places and since small rings are likely to be broken at most once (because the density of breaks is small), the distribution of open chains must scale as

$$
\ell P(\ell) \sim \ell^{-\vartheta+1}
$$

for the parameters of Fig. 10(c), $\vartheta \simeq 1.3$. The additional peaks at $\ell=L, 2 L, \ldots$, are a consequence of our use of periodic boundary conditions. The well-differentiated even and odd distributions seen at small $\ell$ begin to coalesce for 

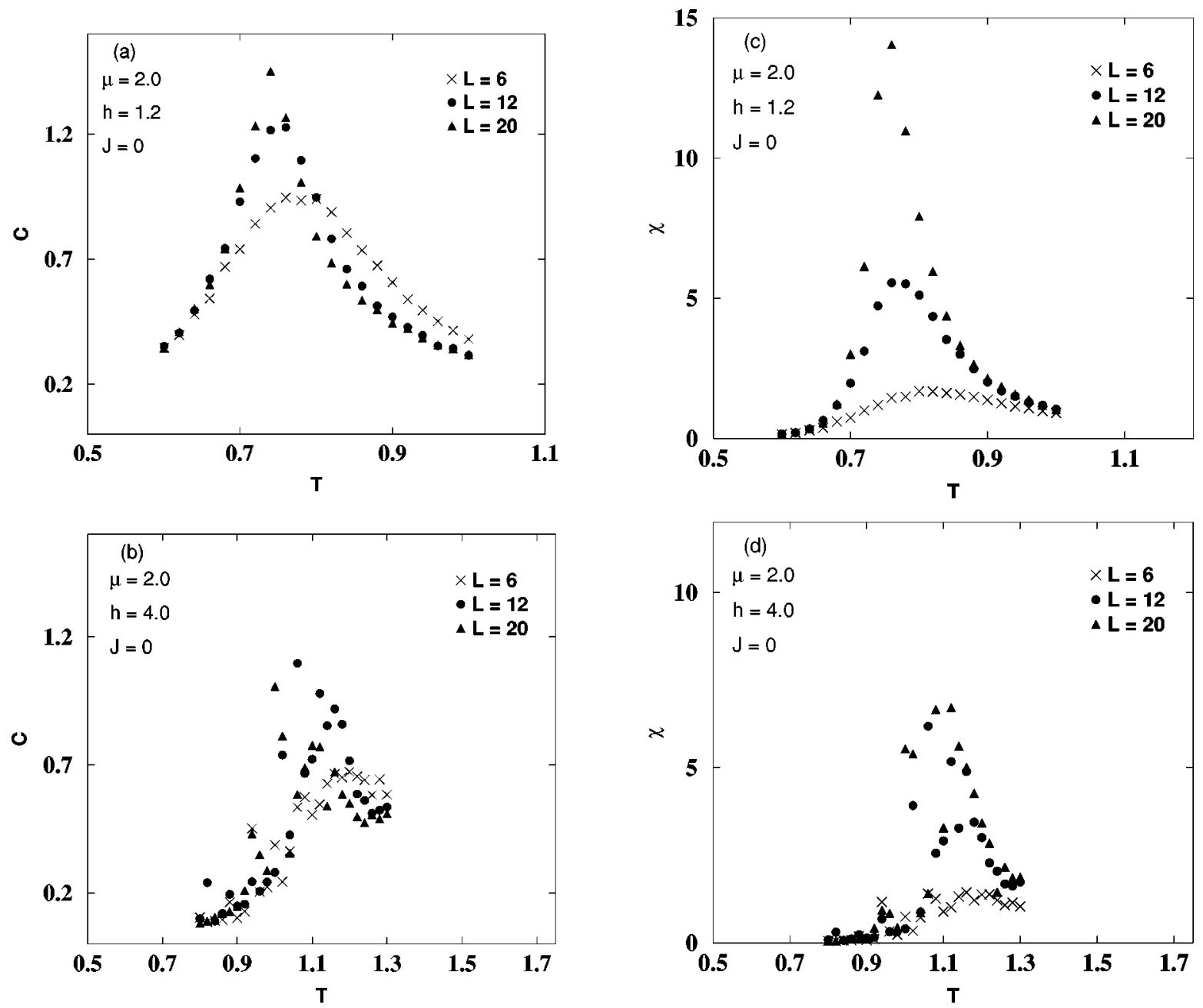

FIG. 7. Specific heat $C$ versus $T$ for $d=2$ as $T$ crosses the transition temperature. The parameters in (a) and (b) are the same as in Figs. 6(a) and 6(b), respectively. The peak sharpens with increasing $L$ in both cases (a) and (b), but the data are distinctly more noisy for the latter. The susceptibility $\chi$ versus $T$ for $d=2$ as $T$ crosses the transition temperature is shown in (c) and (d) for two choices of parameter values. The parameters in (c) and (d) are the same as in Figs. 6(a) and 6(b), respectively. The peak sharpens with increasing $L$ in cases (a) -(d), but the data are distinctly more noisy for (b) and (d).

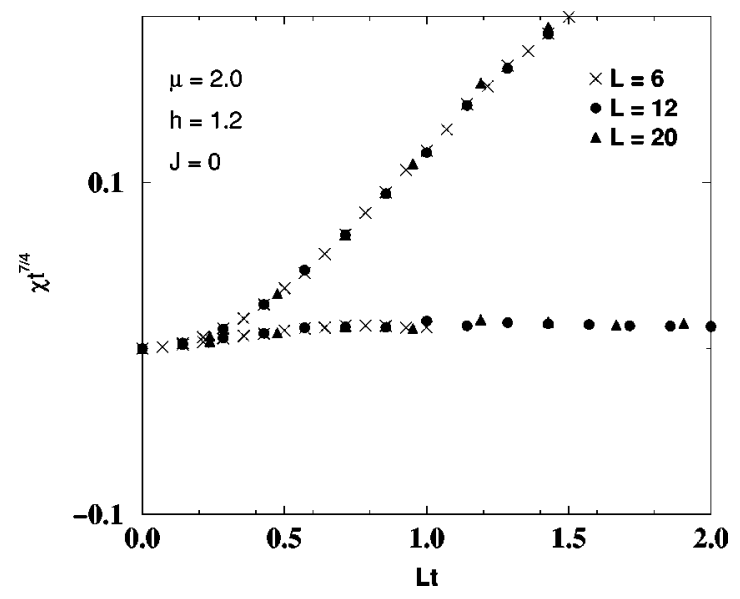

FIG. 8. Finite-size-scaling plot for the susceptibility data of Fig. 7(d) for (linear) system sizes $L=6,12$, and 20. Curves for different values of $L$ collapse onto two scaling curves (one for $T>T_{c}$ and the other for $T<T_{c}$ ). Here we have used the two-dimensional Isingmodel exponents $\gamma=7 / 4$ and $\nu=1$, which characterize, respectively, the divergences of the susceptibility and the correlation length at criticality.

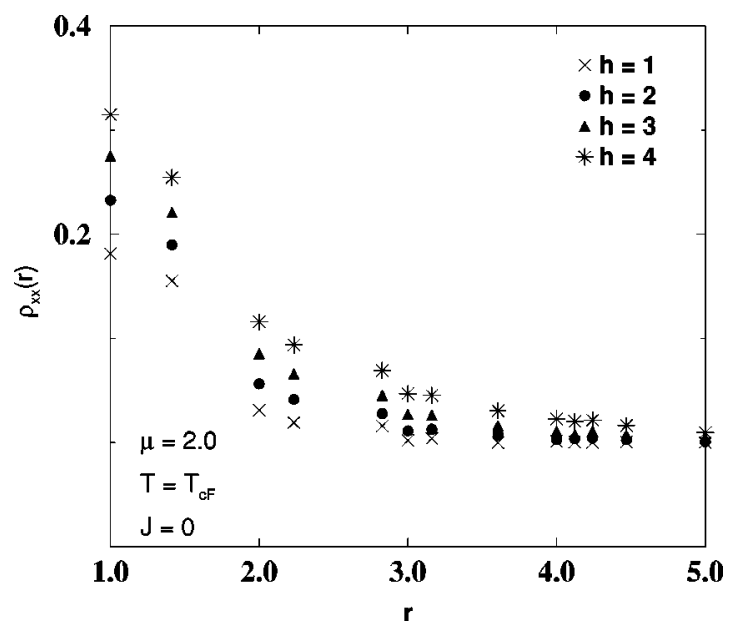

FIG. 9. Angle-averaged (see the text) correlation function $\rho_{x x}\left(x, y, x^{\prime}, y^{\prime}\right) \equiv\left\langle\rho_{x}(x, y) \rho_{x}\left(x^{\prime}, y^{\prime}\right)\right\rangle-\left\langle\rho_{x}(x, y)\right\rangle\left\langle\rho_{x}\left(x^{\prime}, y^{\prime}\right)\right\rangle \quad$ versus the radial coordinate $r$, for $h=1,2,3$, and $4, \mu=2, J=0, L$ $=20$, and $T=T_{c}^{F}$, the transition temperature of the $F$ model. These data indicate clearly that this correlation function decays more and more slowly as we approach the $F$-model limit. 

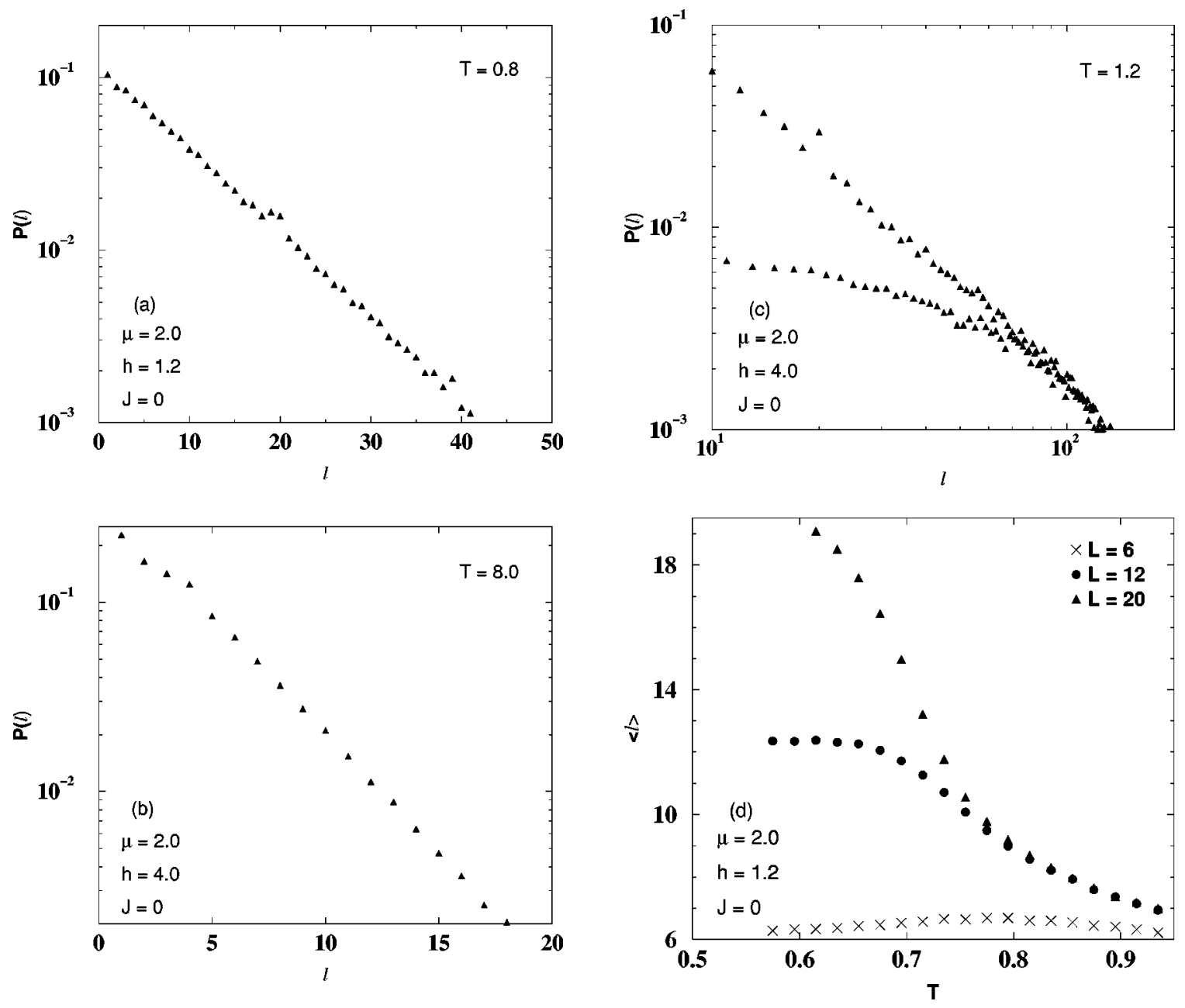

FIG. 10. The distribution of polymer lengths $P(\ell)$ versus $\ell$ for $L=20, T=0.8, \mu=2, h=1.2$, and $J=0$ is shown in $($ a). $P(\ell)$ clearly decays exponentially in this case. The distribution of polymer lengths $P(\ell)$ versus $\ell$ for $L=20$ with the parameters (b) $T=8, \mu=2$, $h=4$, and $J=0$ and (c) $T=1.2, \mu=2, h=4$, and $J=0$ is shown in (b) and (c). Note the clear exponential decay at large $T$. For very large $\ell, P(\ell)$ must decay exponentially; however, for the scales studied here in (c), it shows a power-law decay and different behaviors for chains with an even number of monomers (upper curve) and an odd number of monomers (lower curve) as explained in the text. A plot of the mean polymer length $\ell_{a v}$ versus $T$ for $d=2$ as $T$ crosses the transition temperature for the parameters of Fig. 6(a) is given in (d).

large $\ell$. In related simulations of the $F$ model (whose polymer analog has only closed loops), we have found a similiar power-law behavior in $P(\ell)$ [42]; in this limit it may be possible to obtain the exponent $\vartheta$ analytically.

We expect the power-law behavior of $P(\ell)$ to cross over to an exponential form (i.e., $P(\ell) \sim e^{-\ell / \ell}$ ) for largeenough lattice sizes. To see this, consider a particular chain (of length $\ell$ ) and a configuration of all other chains consistent with this chain. If $\ell$ is large, the probability that the chain is unbroken is an exponentially decreasing function of $\ell$. [For the purposes of establishing an upper bound for $\ell_{0}$, the length at which $P(\ell)$ crosses over to an exponential form, we can consider the chain to be extended (ignoring bends) and disallow adjacent breaks in the chain.] The characteristic length of the distribution is then found to scale as $\ell_{0} \sim e^{2 \beta h}$. We estimate, for $T \simeq 1$ and $h=4$, that $\ell_{0} \simeq 3000$ in units of the lattice spacing, i.e., the crossover to exponential behavior in $P(\ell)$ would show up at these temperatures on lattices with $L>3000$. Note that for $T=8$ [Fig. 10(b)], this crossover length is $\simeq 3$, which is well within our finite lattice size. Though the inclusion of bends, etc., can renor- malize $\ell_{0}$, we expect that this will not alter our bound significantly.

For case (a) Fig. 10(d) shows the average polymer length $\langle\ell\rangle$ versus $T$ for three different lattice sizes. We expect that $\langle\ell\rangle$ displays a mild nonanalyticity at $T_{C}$ that is masked by an analytic background term; we have not tried to extract this nonanalytic part. However, we would like to emphasize that this mild nonanalytic behavior is associated with the ordering transition described above and not with what might be called a strict polymerization transition, i.e., one below which $\langle\ell\rangle \simeq A L$, where $A$ depends on $T$, $\mu$, etc. As far as we can tell from our simulations on finite lattices, such strict polymerization occurs only at $T=0$ in our model with $A$ =1: The ground-state configuration is a stack of circular polymer chains (looping around the lattice in one direction since we use periodic boundary conditions). At any finite temperature, $\langle\ell\rangle$ becomes independent of $L$ for sufficiently large system sizes (clearly this cannot be checked numerically at very low temperatures). In case (b) $P(\ell)$ is very broad, so, for system sizes $L<L_{c},\langle\ell\rangle \gg L$; however, at any $T>0$, once $L>L_{c},\langle\ell\rangle$ must eventually assume an 
$L$-independent value and, as in case (a), strict polymerization occurs only at $T=0$.

We now consider the inclusion of the interpolymer attraction $J$ : Since it is not a symmetry-breaking interaction we expect the Ising-type transition for $J=0$ to persist for small $J>0$; for large $J$ this transition could disappear or perhaps change its order. Our Monte Carlo simulations for $0<J / \epsilon$ $<0.4$ coupled with finite-size scaling analysis indicate that the two-dimensional Ising-type critical behavior persists for small finite $J$.

\section{THREE DIMENSIONS}

The transition in the two-dimensional case is of interest because of the controversy regarding its behavior. However, the three-dimensional case is physically more interesting. In three dimensions our model exhibits a first-order transition in all the regimes of parameter space that we have explored. All quantities, such as the order parameter, internal energy, and $P(\ell)$, change discontinuously at this transition. This result is in agreement with Flory's prediction of a first-order crystallization transition. If the concentration of open ends is very small, we see substantial hysteresis as we cycle $T$ through the first-order transition; the width of this hysteresis loop cannot be reduced significantly given the time scale of our simulations. Equilibration is difficult in this limit for two reasons: (i) The breaking of chains is energetically disfavored and (ii) since our multilink moves conserve the number of links, the simulation effectively becomes a canonical one.

Our simulations in $d=3$ were carried out on lattices of linear dimension $L=8$ and 16 , with $J=0.3$. (It is necessary to include this attractive term to obtain an ordered ground state; otherwise one could, with no energy cost, disorder the aligned state merely by rotating one aligned plane with respect to another.) Most of the data we give here were obtained with $\mu=2$ and $h=1.2$. We have also studied other values of $\mu$ and $h$; we find that the first-order transition remains for all the parameter values we have studied. However, equilibration is hampered at large values of $h$. Our simulation method was similiar to the one we used in $d=2$ and included a defect-removal move every $20 \mathrm{MCS}$. (Note that for $J>0$, the energy required to remove or to add a defect is not $\pm 4 \epsilon$, but also depends on the configurations of neighboring links.) We found that defect removal, though useful, was not as important for equilibration in $d=3$ as in $d=2$. We accumulated data for thermodynamic quantities every 10 MCS typically for $(2-5) \times 10^{4}$ iterations, at each value of $T$ and other couplings.

As in $d=2$ we define suitable order parameters to characterize the broken-symmetry state at low temperatures. These are

$$
\begin{aligned}
& M_{x y}=\left(N_{x}-N_{y}\right) / N_{\text {links }}, \\
& M_{y z}=\left(N_{y}-N_{z}\right) / N_{\text {links }}, \\
& M_{x z}=\left(N_{x}-N_{z}\right) / N_{\text {links }},
\end{aligned}
$$

where $N_{x}, N_{y}$, and $N_{z}$ are the numbers of occupied links in the $x, y$, or $z$ directions, respectively, and $N_{\text {links }}$ is the number of links occupied in the fully ordered state. The three associated susceptibilities are obtained as in $d=2$.

The transition is clearly first order, with the orderparameter jump [Fig. 11(a)] typically $0.6-0.7$. Triangles and crosses indicate heating and cooling runs, respectively; the absence of hysteresis indicates proper equilibration. For large $h$ there is substantial hysteresis: In this limit the cost of open ends is very high and so closed chains predominate and our single-link updates are ineffective for equilibration. Figures 11(b) and 11(c) show, respectively, the specific heat and susceptibility of our model in $d=3$ for $L=16$. The peaks in both cases sharpen rapidly with increasing $L$ (they should be $\delta$ functions for $L=\infty$ ). In Fig. 11(d) we plot the densities of bends $N_{b}$ and vacancies $N_{v}$ and in Fig. 11(e) the density of open ends $N_{o}$, which are, as expected, discontinuous at the transition [in contrast with Figs. 6(e) and 6(f)].

Figures 12(a) and 12(b) show the evolution of the polymer length distribution $P(\ell)$ with $T: P(\ell)$ decays exponentially both above and below the transition; however, there is a sharp discontinuity in the decay length across the transition as can be seen from the intercepts on the $\ell$ axis. Figure 12(c) shows the sharp drop in the average length $\ell_{a v}$ at the transition. For the smaller lattice size there is an increase in $\ell_{a v}$ at the transition. Indeed, for any finite $L, \ell_{a v}$ is not a monotonic function of $T$ : At $T=0, \ell_{a v}=L$, but at low $T>0$ it increases to higher values, peaking at a temperature below the transition temperature for orientational ordering.

\section{GLASS FORMATION}

In most experimental situations (when crystallization is obtained by decreasing $T$ ), it is necessary to cool the melt slowly; otherwise the system drops rapidly out of equilibrium. The resulting state is a glass to the extent that it has only short-ranged order and typically does not evolve significantly over experimental time scales. Slow heating of this glass yields transitions to a crystal and eventually the melt, which show up as exothermic and endothermic peaks, respectively, in differential scanning calorimetry. Furthermore, temporal autocorrelations in such glassy states usually show stretched-exponential relaxation $[3,4]$. Recent studies $[8,13]$ have also begun to explore glass (or gel) formation in systems of living polymers.

Given the ubiquity of glass formation in experimental polymeric melts, it is natural to ask if our model can yield such glassy behavior. We show below that it does both on quenching instantaneously from high to low $T$ and on cooling at finite rates. The resulting vitreous states share some properties with experimental polymeric glasses. Such behavior obtains in our model both in $d=2$ and $d=3$; we concentrate on the latter since it is more relevant for experiments. We have given a brief account of glass formation in our model elsewhere [16]. We summarize these findings here so that all results pertaining to our model for living polymeric melts are available together. We also discuss the relation of our work with that of other workers [2,26-29] in Sec. V.

When we quench our system from the disordered phase at high $T$ to the ordered phase at a temperature $\simeq T_{c} / 2$ in $d$ $=3$, we find that, if $h$ is small, equilibration is rapid and the disordered state evolves to the ordered one typically over 100-1000 MCS for $L=16$. Evolution to the ordered state 

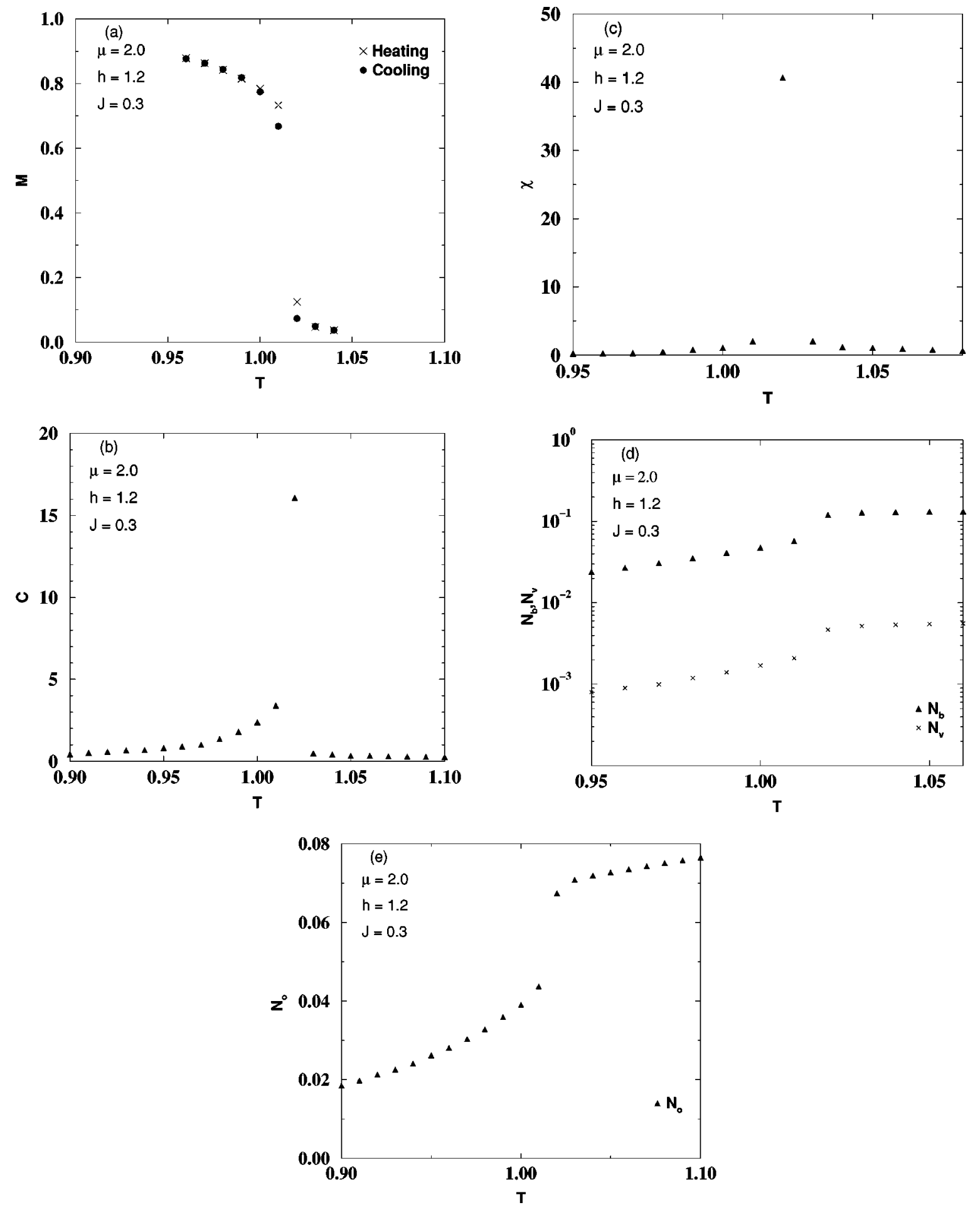

FIG. 11. (a) Order parameter $M$ versus $T$ for our three-dimensional model. The first-order nature of the crystallization transition in $d$ $=3$ is clearly manifested in the jump of $M$ at the transition temperature $\left(T_{c} \simeq 1.02\right)$. A cooling and a heating run are shown for a system of (linear) size $L=16$. The parameter values are $\mu=2, h=1.2$, and $J=0.3$. (b) Specific heat per link $C$ versus $T$ for $d=3$ and the parameters of (a). The peak should be a $\delta$ function, but is smeared by finite-size effects. (c) Susceptibility per link $\chi$ versus $T$ for $d=3$ and the parameters of (a). (d) Temperature dependence of the densities of bends and vacancies $N_{b}$ and $N_{v}$ and (e) density of open ends $N_{o}$ for $d$ $=3$ and the parameters of (a).

becomes increasingly sluggish as $h$ is increased. For example, for $h=5.5$, we see essentially no increase in the order parameters $\left(\simeq 0\right.$ before the quench) over $5 \times 10^{5}$ iterations and the resulting configurations are completely disordered [Fig. 1(a)].

Large values of $h(>3)$ suppress local rearrangements, yield completely disordered glasses [Fig. 1(a)], and logarithmically slow temporal evolution of, e.g., $E$, especially for our deepest quenches $\left[10^{3} \leqslant t \leqslant 2 \times 10^{5}\right.$ MCS in Fig. 13(a)]; we have obtained similar, though more noisy, data for the vacancy concentration $N_{v}$ and order parameters. At intermediate $h(1.5<h<3)$ we find different vitreous states, which 

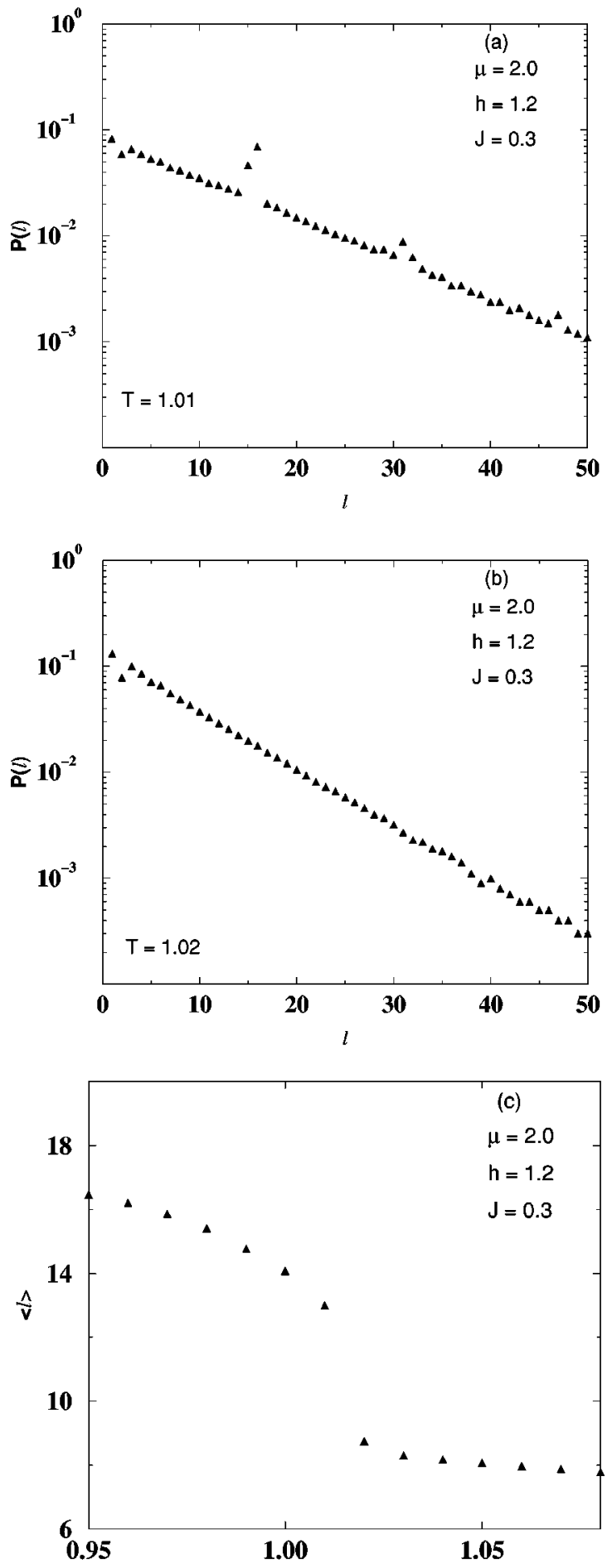

FIG. 12. Distribution of polymer ${ }^{\mathbf{T}}$ lengths $P(\ell)$ versus $\ell$ for $d$ $=3$, the parameters of Fig. 11(a), and (a) $T=1.01$ and (b) $T$ $=1.02$. $P(\ell)$ decays exponentially, but has subsidiary peaks at values of $\ell$ commensurate with the lattice dimension. Note that the distribution at $T=1.02$ is much narrower than the one at $T=1.01$, indicating a discontinuous change in $\ell_{a v}$ at this first-order transition. The mean polymer length $\ell_{a v}$ versus $T$ for $d=3$ and the parameters of Fig. 11(a) is shown in (c). Note the sharp jump in $\ell_{a v}$ at the crystallization transition.

we term lamellar glasses [the one-dimensionally disordered stacking of ordered layers of polymers shown in Fig. 1(b)]; the quenched system evolves to such a lamellar glass in a time $\tau_{l g}$; order parameters saturated to values between 0 (the
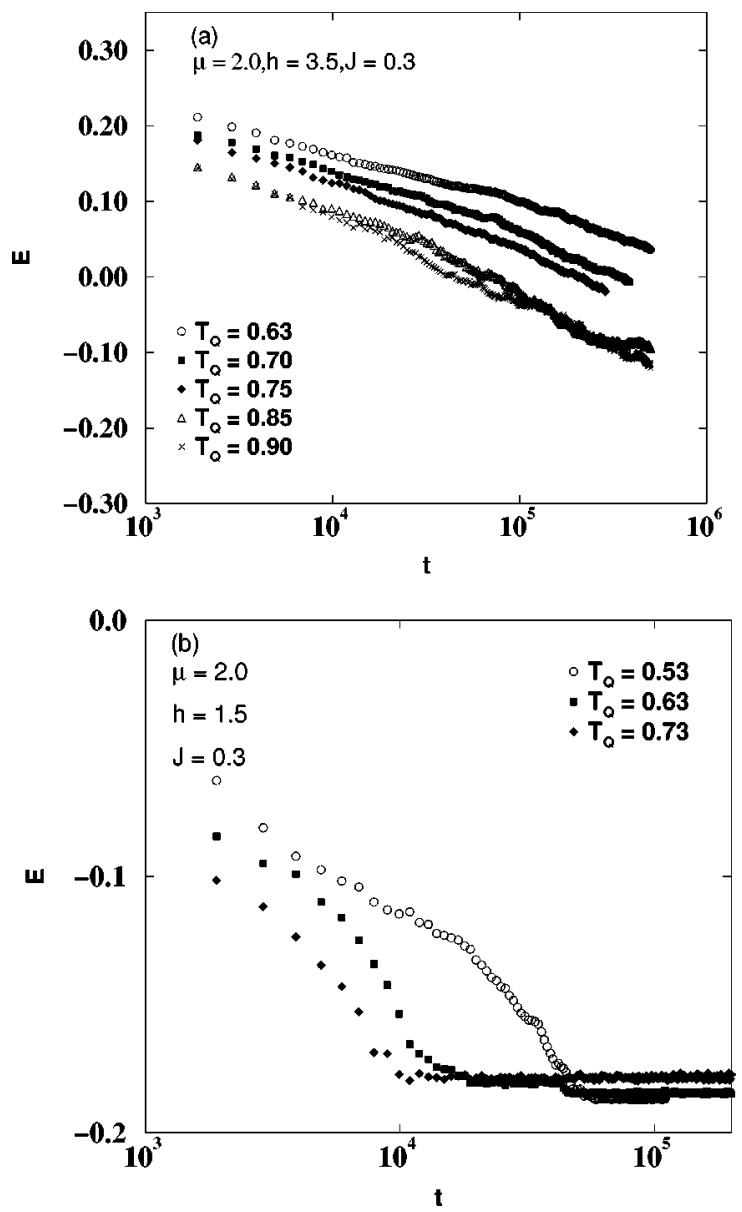

FIG. 13. Energy $E$ versus time $t$ after a quench to $T=T_{Q}$ for $L=16$ showing (a) a logarithmic decay at large $h$ and (b) and a somewhat faster decay at intermediate $h$. In (b) the system eventually becomes a lamellar glass and $E$ does not evolve over our $t$ range.

high- $T$ value) and 1 (the perfect crystal). Our data for the time evolution of the internal energy $E$ [Fig. 13(b)] are consistent with $\tau_{l g} \sim \exp (2 \beta h)$. After they are formed, these lamellar glasses do not evolve substantially even if annealed for $\simeq 10^{6}$ MCS since a large number [at least $O\left(L^{2}\right)$ ] of cooperative local updates are required to align all planes. All our glassy states yield order-parameter autocorrelation functions that are slowly decaying exponentials for shallow quenches; deeper ones yield decays that are so slow that we cannot obtain reliable fits.

Our model has other interesting glass-related features. (i) It falls out of equilibrium not only when it is quenched, but also when it is cooled at a finite rate, as shown in Fig. 14(a). Thus we have studied it via a [16] Monte Carlo analog of scanning calorimetry [Fig. 14(b)] and differential scanning calorimetry, all of which are in qualitative accord with the behavior of real polymeric glasses [4]. (ii) It shows a glasscrystal transition on lowering $h$, presumably because this eases the frustration in the disordered network obtained on quenching. This transition is seemingly continuous as can be seen from Fig. 15; however, it is not related to an underlying equilibrium phase transition as we show below.

In our studies of vitrification we use simple cubic lattices of size $16^{3}$. The Monte Carlo algorithm is the same as in our equilibrium studies (Sec. III), so we do not conserve mono- 

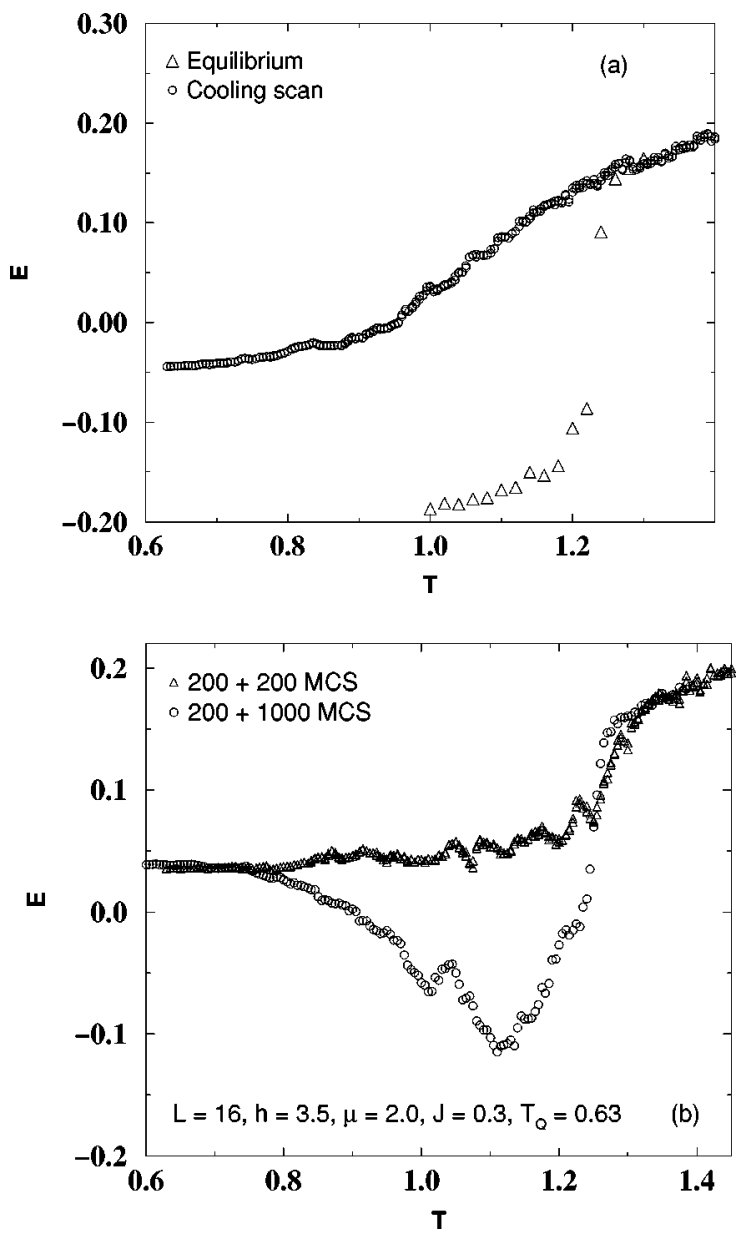

FIG. 14. (a) Evolution of $E$ with temperature $T$ on steady cooling (circles) to $T<T_{c}$ at large $h[=3.5$ here with annealing and recording times (see the text) $\left.t_{a}=t_{r}=100\right]$ and in equilibrium (triangles). (b) $E$ versus $T$ for our Monte Carlo scanning calorimetry (see the text) after a quench to $T_{Q}=0.63$. We use two heating rates indicated by triangles $\left(t_{a}=200\right.$ and $\left.t_{r}=200\right)$ and circles $\left(t_{a}=200\right.$ and $\left.t_{r}=1000\right)$. At the slower heating rate (circles), the glass transforms more effectively into a crystal (the flat minimum in the curve with $E \simeq-0.13$; for the ordered crystal $E \simeq-0.2$ ) before it melts eventually into the disordered high- $T$ phase.

mers, vacancies, or order parameters. Nonetheless, glasses are formed as mentioned above. We present some details of our calculations below.

For studying the quench we use a high-temperature ( $T$ $=10$ ) configuration, with $h=1.2, \mu=2$, and $J=0.3$. This yields a low density of short chains $\left(\ell_{a v} \sim 4\right)$. We quench this initial configuration in one step to $T_{c} / 2$ over a range of parameter values (we vary $\mu$ and $h$ and use the $T_{c}$ appropriate for the parameter values). We then track the temporal evolution of the internal energy per link $E$, the order parameter $M$, and the average number of vacancies $N_{v}$. We have checked that different initial configurations, obtained with different sequences of random numbers or by starting at $T$ $=\infty$ (i.e., adding and removing links from an initially empty lattice at random but respecting self-avoidance), yield the same qualitative behaviors; the data we present here are for a typical run. We compute $E(t)$, etc., ( $t$ in MCS) by averaging over 20 measurements, separated by $10 \mathrm{MCS}$ each and centered at $t$. We evolve the system for $t \simeq(3-6)$ $\times 10^{5}$ MCS.

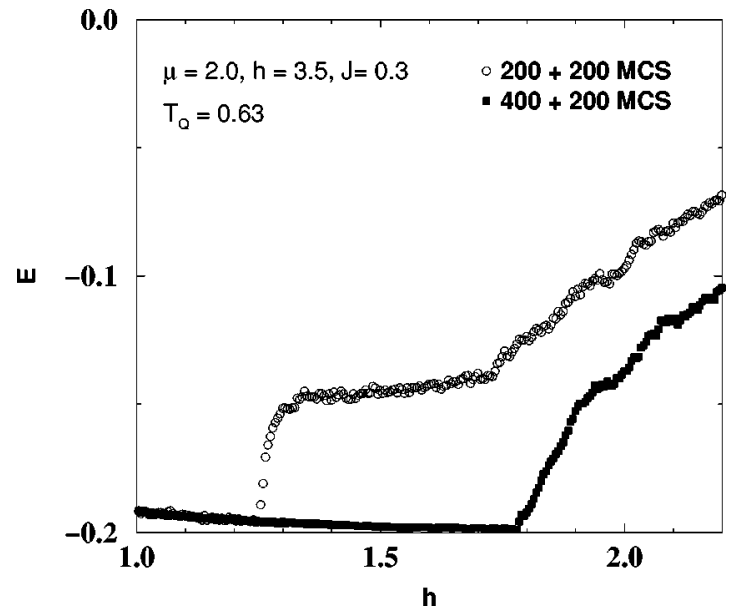

FIG. 15. $E$ versus the frustration parameter $h$ at two scanning rates (see the text) with $t_{r}=200$, but $t_{a}=200$ (open circles) and 400 (filled squares). As $h$ is lowered from 3.5, its value when we quench to $T_{Q}=0.63$, an apparently continuous glass-crystal transition occurs and $E$ flattens out. The value of $h$ at the transition depends on the scanning rate.

By annealing quenched configurations at different $T<T_{c}$ we have calculated autocorrelation functions such as $\mathcal{A}_{x}(t)$ $\equiv\left(1 / L^{3}\right) \Sigma_{i}\left[\left\langle\rho_{x}\left(i, t_{0}=0\right) \rho_{x}\left(i, t_{0}=t\right)\right\rangle-\left\langle\rho_{x}\left(i, t_{0}\right)\right\rangle^{2}\right]$ and its $y$ and $z$ analogs. We find they fit exponential forms with large autocorrelation times. These times increase as $h$ is increased or as $T$ is decreased. For $h=3.5$ and $T=0.7$, these autocorrelation times are $\simeq 500$ MCS. Unfortunately, at lower $T$ or higher $h$, these decays are so slow that we cannot get good data for meaningful fits. Thus our study leaves open the possibility of nonexponential large- $t$ behaviors in our low-temperature glassy states. Note, though, that the logarithmic decays [e.g., Fig. 13(a)] displayed by the glasses in our model are reminiscent of those found in disordered systems. Our model has no random couplings, but the disorder arises dynamically via the quench or cooling at a finite rate, as in conventional glasses. Also, the slow decrease of $N_{v}$ with $t$ is analogous to the volume contraction obtained when polymeric glasses [43] are aged. This is ascribed to decreasing free volume in some theories [25].

To study glass formation on steady cooling, at a rate not slow enough for equilibration, we begin with equilibrated configurations at $T=1.5$ and lower $T$ in steps of 0.005 . We divide the time $t$ (MCS) spent at a given set of parameters (such as $T$ in Fig. 14 and $h$ in Fig. 15) into an annealing time $t_{a}(\simeq 200-1000 \mathrm{MCS})$, during which we do not collect data, and a recording time $t_{r}(\simeq 200-1000 \mathrm{MCS})$, in which we accumulate data for averages every $10 \mathrm{MCS}$. Cooling or heating rates follow simply from the value of $t \equiv t_{a}$ $+t_{r}$ shown. The glasses we obtain thus are similar to those resulting from our quenches: For small $h<1.5$ our system can be supercooled just a little before it crystallizes, but, in the intermediate range $1.5<h<3, E$ drops with $T$, though not as sharply as in equilibrium [Fig. 2(a)] and the system forms a lamellar glasses. For large $h>3$ [Fig. 14(a)] a completely disordered configuration is formed, but with slightly larger ordered patches than for an instantaneous quench.

Our Monte Carlo analog of scanning calorimetry yields successive glass-crystal and crystal-liquid transitions when 
we heat our glasses steadily. The scanning proceeds as follows: We quench the system from $T=10$ to $T_{c} / 2$, anneal it for $6 \times 10^{5} \mathrm{MCS}$, and then increase $T$ at $10^{-6}$ per MCS. Here too the intermediate- $h$ regime yields a lamellar glass, but is not very interesting since it melts directly to the disordered phase. For large $h$ we find that, on heating, the glass transforms into a crystal; on further heating, this crystal melts into the disordered phase. These transformations are mirrored in the behavior of $E$ : It drops sharply at the glasscrystal transition and then increases rapidly at the crystalmelt transition [Fig. 14(b)]. The temperature range over which the crystal appears increases with decreasing heating rate and for slow heating rates [circles in Fig. 14(b)]; at least the crystal-melt transition appears to be discontinuous. The analog of an experimental differential-scanning-calorimetry plot [4] can be obtained through the derivative $d E / d T$. As can be seen from Fig. 14(b), such a derivative will show both an exotherm and a sharp overshoot, as is often seen in rapidly cooled, annealed, and slowly reheated glasses $[3,4]$. The exotherm arises because of relaxation out of a high fictivetemperature state formed by the quench. Of course, the crystal-melt transition resembles equilibrium melting. More complicated transitions occur in some of our scans: We have seen the glass transform to a crystal that reforms into a glass at a slightly higher temperature; this glass then becomes a crystal that melts to the disordered phase.

The energy cost for open ends $h$ plays a crucial role in slowing down the kinetics in our model. By contrast, we have checked that, for large $h$, it plays a minor role in determining the equilibrium $T_{c}$. This arises because of the large energy barrier for the removal of a link $(\sim h$, for large $h$ ) and, along with the self-avoidance constraint, leads to a sort of frustration that results in vitrification. This frustration can clearly be reduced by lowering $h$ and results in a glasscrystal transition: Specifically, we prepare glassy configurations by annealing quenched configurations for 6 $\times 10^{5} \mathrm{MCS}$ at $T_{c} / 2$ and large $h(h=3.5$ in Fig. 15). We then decrease $h$ in steps of $5 \times 10^{-3}$. This gives us an apparently continuous transition: $E$ decreases monotonically and smoothly to its mean value in the crystal. However, we wish to emphasize that, in our model there is no equilibrium transition underlying this frustration-driven transition, in contrast to some scenarios for the glass transition [26]. First, this transition is not reversible, for there is no crystal-glass transition when $h$ increases; second, the value of $h$ at this transition depends on the rate at which we change $h$ : Scans slower than those of Fig. 15 make the transition disappear, for the crystal is eventually stabilized.

\section{CONCLUSION}

We have presented a lattice model for the transition to an ordered state in melts of semiflexible, living polymers and studied its statistical mechanics in two and three dimensions via Monte Carlo simulations and the analysis of exactly solvable limits. We have shown that in two dimensions strong crossover effects arise because of the proximity to the $F$-model fixed line and can lead to very large correlation lengths and slow relaxation. We have demonstrated the existence of an Ising-type transition separating the ordered and disordered phases for the two-dimensional square lattice.
Though the universality class of this transition might be different for continuum systems, it would be interesting to look experimentally for continuous transitions to crystalline order in two-dimensional melts of living polymers. In three dimensions the transition is first order.

Our study shows how an interplay of semiflexibility, selfavoidance, and the energy cost for breaking chains leads to the vitrification of a melt of living polymers. Furthermore, our model is a good testing ground for theories of the glass transition since frustration can be tuned easily. In a more general context, our model can be thought of as a spin (or lattice-gas) model without quenched disorder that exhibits a transition to a glassy state at low temperatures [45]. Also, it would be interesting to see whether lamellar glasses form in real polymeric melts or are merely an artifact of our lattice model.

Systems of living polymers have also been modeled by independent-monomer-state models $[9,23,24]$. As we have mentioned earlier, there are some important differences between these and our model for living polymers. The IMS models admit states that are disallowed in our model. These differences are perhaps more important in two than in three dimensions because of the relation of our model to vertex models such as the $F$ model. This might well explain why some IMS models $[9,23]$ yield second- and first-order boundaries meeting at a tricritical point [23] whereas our model yields an Ising-type continuous transition. In three dimensions both our model and the IMS models [9] agree qualitatively insofar as both yield first-order melt-crystal transitions at which thermodynamic functions and polymer-length distributions change discontinuously. To the best of our knowledge, vitrification has not been studied in the context of IMS models.

Our model (and the IMS models) can be thought of as a grand-canonical generalization of the Flory model, for it permits variable monomer densities and chain lengths governed by an equilibrium distribution. In the models studied by Flory and Baumgärtner [1,19-21] chain lengths are fixed and cannot fluctuate; also no ring polymers are allowed. To the extent that these differences do not matter, our analysis provides an explanation (because of slow equilibration arising from the proximity of the $F$-model critical line) for the considerable controversy that has surrounded studies of the Flory and related models in two dimensions [1,15,18-20,22]. In three dimensions our results are consistent with Flory's prediction of a first-order crystallization transition.

Most experiments are done on fixed-length polymeric systems without ring polymers. These are better described by the Flory model than by our model. Our results should be more directly applicable to systems of living polymers that have been attracting attention over the past decade $[6,8,10$ 14]. Important issues include [13] an elucidation of the conditions under which living polymeric systems form glasses. We believe our work is the first comprehensive numerical study of this issue in a model for living polymers. We hope our work stimulates experimental studies of glasses and gels in melts of living polymers, which are just beginning to be studied $[8,13]$. However, some care must be exercised in using our lattice-model results to interpret continuum experiments.

Perhaps the earliest theoretical study of polymeric glasses 
was that of Gibbs and Di Marzio [2]. They used the configurational entropy calculated via Flory's mean-field approximation [1] and extrapolated below the mean-field transition temperature $T_{m f}$ to obtain a second-order glass transition at which the entropy vanished, but a finite concentration of gauche bonds remained. Their work relies on two assumptions: (i) that Flory's approximation is valid, and (ii) that such an extrapolation is meaningful. The second assumption is especially questionable for it is not clear that this vitrification is associated with an underlying (thermodynamic) continuous transition or results because of very slow kinetics arising from constraints such as excluded volume, which becomes important at high densities: For example, simulations of two-component mixtures of hard spheres have obtained glassy behavior in assemblies of as few as 32 particles without the divergence of any correlation length [44]. All our work shows that, at least for the model system of living polymers we study, vitrification arises because of slow kinetics and not an underlying (equilibrium) continuous transition.

In recent Monte Carlo simulations of glasses in a twodimensional lattice model for a conventional polymeric melt, Ray and Binder $[28,29]$ find glassy states with nonexponential decays of autocorrelation functions at low $T$. However, their model has an equilibrium transition only at $T=0$, so it cannot yield scanning-calorimetric plots such as ours, nor does it have a simple frustration parameter that can be varied. However, since their simulation conserves the number of monomers, they can obtain diffusion constants that we cannot. Our model and the spin-facilitated model of Fredricksen and Andersen [27] have interesting connections, for, in both, relaxation at low temperatures occurs via highly cooperative moves. However, our model has the advantage that it yields both equilibrium freezing and glass formation. Of course, to model glass formation in real polymeric melts, we should, ideally, use a continuum description and enforce the relevant conservation laws. These conservation laws will, in general, lead to longer equilibration times and slower relaxation than in our model, in which no quantity is conserved.

\section{ACKNOWLEDGMENTS}

We thank J. Banavar, M. Barma, C. Dasgupta, D. Dhar, S. Dhar, C. Jayaprakash, T. V. Ramakrishnan, S. Ramaswamy, M. Rao, and R. Rao for stimulating discussions, UGC, CSIR, and DST (India) for support, and SERC (IISc Bangalore) for computational resources. One of us (G.I.M.) is grateful for support from NSERC of Canada.
[1] P. J. Flory, Proc. R. Soc. London, Ser. A 234, 60 (1956); 234, 73 (1956).

[2] J. H. Gibbs and E. A. DiMarzio, J. Chem. Phys. 28, 373 (1958); E. A. DiMarzio and J. H. Gibbs, ibid. 28, 807 (1958).

[3] I. M. Hodge, J. Non-Cryst. Solids 169, 211 (1994).

[4] S. Z. D. Cheng, Z. Q. Wu, and B. Wunderlich, Macromolecules 20, 2802 (1987).

[5] M. S. Turner and M. E. Cates, J. Phys. (France) 51, 307 (1990).

[6] G. Porte, J. Marignan, P. Bassereau, and R. May, J. Phys. (France) 49, 511 (1988).

[7] Even though surfactant concentrations may be low, the system is effectively dense if the long-ranged Coulomb interaction between (ionic) micelles is not screened by an electrolyte.

[8] B. K. Mishra, S. D. Samant, P. Pradhan, S. B. Mishra, and C. Manohar, Langmuir 9, 894 (1993); P. S. Goyal, R. Chakravarthy, B. A. Dasannacharya, J. A. E. Desa, V. K. Kelkar, C. Manohar, S. L. Narasimhan, K. R. Rao, and B. S. Valaulikar, Physica B 156-157, 471 (1989).

[9] A. Milchev and D. P. Landau, Phys. Rev. E 52, 6431 (1995).

[10] J. C. Wheeler, S. J. Kennedy, and P. Pfeuty, Phys. Rev. Lett. 45, 1748 (1980); S. J. Kennedy and J. C. Wheeler, J. Chem. Phys. 78, 953 (1983).

[11] G. Faivre and J. L. Gardissat, Macromolecules 19, 1988 (1986).

[12] K. M. Zheng and S. C. Greer, Macromolecules 25, 6128 (1992); K. M. Zheng, S. C. Greer, L. R. Corrales, and J. RuizGarcia, J. Chem. Phys. 98, 9873 (1993); A. P. Andrews, K. P. Andrews, S. C. Greer, F. Boué, and P. Pfeuty, Macromolecules 27, 3902 (1994); S. S. Das, A. P. Andrews, and S. C. Greer, J. Chem. Phys. 102, 2951 (1995).

[13] S. C. Greer, Comput. Mater. Sci. 4, 334 (1995).
[14] F. Oozawa and S. Asakura, Thermodynamics in the Polymerization of Proteins (Academic, New York, 1975).

[15] G. I. Menon, R. Pandit, and M. Barma, Europhys. Lett. 24, 253 (1993).

[16] G. I. Menon and R. Pandit, Phys. Rev. Lett. 75, 4638 (1995).

[17] J. F. Nagle, Proc. R. Soc. London, Ser. A 337, 569 (1974).

[18] P. D. Gujrati and M. Goldstein, J. Chem. Phys. 74, 2596 (1981).

[19] A. Baumgärtner, J. Phys. A 17, L971 (1984).

[20] A. Baumgärtner, J. Chem. Phys. 84, 13 (1986).

[21] A. Baumgärtner and D. Y. Yoon, J. Chem. Phys. 79, 521 (1983).

[22] H. Saleur, J. Phys. A 19, 2409 (1986).

[23] G. F. Tuthill and M. V. Jaric, Phys. Rev. B 31, 5 (1985); 31, 2981 (1985).

[24] A. Milchev, Polymer 34, 362 (1993).

[25] M. H. Cohen and G. S. Grest, Phys. Rev. B 20, 1077 (1979).

[26] J. P. Sethna, Europhys. Lett. 6, 529 (1988).

[27] G. H. Fredrickson and H. C. Andersen, Phys. Rev. Lett. 53, 1244 (1984).

[28] P. Ray and K. Binder, J. Phys.: Condens. Matter 5, 5731 (1993); J. Non-Cryst. Solids 172-174, 204 (1994).

[29] P. Ray, J. Baschnagel, and K. Binder, J. Phys.: Condens. Matter 5, 5731 (1993); P. Ray and K. Binder, J. Non-Cryst. Solids 172-174, 204 (1994); Europhys. Lett. 27, 53 (1994).

[30] E. M. Lieb and F. Y. Wu, in Phase Transitions and Critical Phenomena, edited by C. Domb and M. S. Green (Academic, London, 1972), Vol. 1, p. 331.

[31] F. T. Wall and F. Mandel, J. Chem. Phys. 63, 11 (1975).

[32] M. E. Fisher and J. Stephenson, Phys. Rev. 132, 1411 (1963).

[33] Along the first-order line separating the dimer and vacancy phases, an infinity of "dimer-vacancy" phases have the same 
energies and coexist with them. These phases are characterized by a configuration of dimers and vacancies arranged in such a way that the dimers do not violate the self-avoidance constraint; they do not occur at finite $T$ in the regions we explore.

[34] M. Blume, Phys. Rev. 141, 517 (1966); H. W. Capel, Physica (Amsterdam) 32, 966 (1966).

[35] T. Blum and Y. Shapir, J. Phys. A 23, L511 (1990).

[36] C. Fan and F. Y. Wu, Phys. Rev. B 2, 723 (1970).

[37] R. J. Baxter, Exactly Solved Models in Statistical Mechanics (Academic, San Diego, 1982).
[38] R. J. Baxter, Phys. Rev. B 1, 2199 (1970).

[39] See, e.g., Monte Carlo Methods in Statistical Physics, edited by K. Binder (Springer-Verlag, Berlin, 1979).

[40] S. Samuel, J. Math. Phys. 21, 2806 (1980).

[41] J. L. Black and V. J. Emery, Phys. Rev. B 23, 429 (1981).

[42] G. I. Menon and R. Pandit (unpublished).

[43] H.-H. Song and R.-J. Roe, Macromolecules 20, 2723 (1987).

[44] C. Dasgupta, A. V. Indrani, S. Ramaswamy, and M. K. Phani, Europhys. Lett. 15, 307 (1991).

[45] J. P. Bouchard and M. Mezard, J. Phys. I 4, 1109 (1994). 